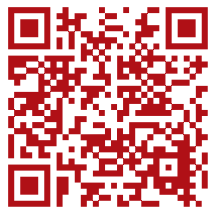

\title{
Diferencias anatomoquirúrgicas de ritidectomía supra-high SMAS vs ritidectomía con plicatura del SMAS en tres vectores
}

\author{
Surgical anatomical differences of SMAS supra-high rhytidectomy \\ vs rhytidectomy with plication of the SMAS in three vectors \\ Dr. David Roland De-Rungs-Brown,* Dr. Martín Manzo-Hernández,* \\ Dr. Adolfo Zamora-Madrazo, * Dr. Armando Martínez-Guzmán, § Dr. Eduardo Alegre-Tamez*
}

Palabras clave: Ritidectomía, sistema musculoaponeurótico superficial, plicatura, ritidosis facial, técnica quirúrgica.

Keywords: Rhytidectomy, superficial musculoaponeurotic system, plication, facial rhytidosis, surgical technique.

\footnotetext{
${ }^{*}$ Cirujano plástico y reconstructivo. Servicio de Cirugía Plástica y Reconstructiva, «American British Cowdray Medical Centern.

\$ Médico cirujano. Universidad Panamericana. $\S$ Cirujano plástico y reconstructivo adscrito al Servicio de Cirugía Plástica y Reconstructiva del Hospital General de México «Dr. Eduardo Liceaga».

Recibido:

04 agosto 2020

Aceptado:

20 enero 2021
}

\section{RESUMEN}

Se han proporcionado descripciones para múltiples técnicas de ritidectomía, unas con mayor manipulación de los tejidos y en consecuencia mayor duración de la cirugía, una recuperación más lenta y mayor probabilidad de complicaciones relacionadas con el procedimiento. En el presente artículo se describe la anatomía patológica de dos técnicas de ritidectomía ampliamente utilizadas: supra-high SMAS y plicatura de SMAS. De manera retrospectiva se comparó la satisfacción estética, el tiempo quirúrgico, la velocidad de recuperación y la probabilidad de complicaciones asociadas en su seguimiento. Se realizó un estudio longitudinal, comparativo, observacional, retrospectivo en 19 casos de plicatura de SMAS y 20 controles de supra-high SMAS. La homogenización de los datos se analizó con las pruebas $\chi^{2}$ y Fisher y utilizamos la razón de momios (OR, IC 95\%) como medida de asociación entre la técnica quirúrgica utilizada y la probabilidad de presentar complicaciones. No hubo diferencia estadísticamente significativa entre la técnica utilizada y la probabilidad de presentar una satisfacción estética $\geq 80 \%, \mathrm{OR}=0.93$ (IC 95\%, 0.22-1.42). Tampoco hubo diferencia estadísticamente significativa entre la técnica utilizada y la probabilidad de no presentar complicaciones postoperatorias, $\mathrm{OR}=2.13$ (IC $95 \%, 0.34$ 13.24). Tanto la ritidectomía supra-high SMAS como la plicatura de SMAS son técnicas de ritidectomía previamente conocidas y ampliamente utilizadas. No existen diferencias en cuanto a satisfacción estética ni en la probabilidad de presentar complicaciones; sin embargo, los pacientes con plicatura de SMAS se operan en menor tiempo quirúrgico y se recuperan de manera más pronta.

\section{ABSTRACT}

Descriptions have been provided for multiple rhytidectomy techniques, some with greater manipulation of the tissues and; therefore, longer surgical time, slower recovery and greater probability of complications related to the procedure. We describe the pathological anatomy of two widely used rhytidectomy techniques: supra-high SMAS and SMAS plication. Next, we compare aesthetic satisfaction retrospectively, intraoperative duration, speed of recovery, and probability of related complications during follow-up. We carried out a retrospective, comparative, observational longitudinal study in 19 cases of SMAS plication and 20 supra-high SMAS controls. The homogenization of the data was analyzed with the $\chi^{2}$ and Fisher tests, and we used the Odds Ratio (OR, 95\% CI) as a measure of association between the surgical technique used and the probability of having complications. There was no statistically significant difference between the technique used and the probability of presenting an aesthetic satisfaction $\geq 80 \%$, OR = 0.93 (95\% CI, 0.22-1.42). There was also no statistically significant difference between the technique used and the probability of not having postoperative complications, $O R$ $=2.13$ (95\% CI, 0.34-13.24). Both the supra-high SMAS rhytidectomy and SMAS plication are previously known and widely used rhytidectomy techniques. There are no significant differences in terms of aesthetic satisfaction or in the probability of having complications; however, the patients with SMAS plication were operated in a shorter time and recovered more quickly.
Citar como: De-Rungs-Brown DR, Manzo-Hernández M, Zamora-Madrazo A, Martínez-Guzmán A, Alegre-Tamez E. Diferencias anatomoquirúrgicas de ritidectomía supra-high SMAS vs ritidectomía con plicatura del SMAS en tres vectores. Cir Plast. 2021; 31 (1): 4-17. https://dx.doi.org/10.35366/101081 


\section{INTRODUCCIÓN}

$\mathrm{P}$ ara poder determinar las diferentes técnicas quirúrgicas para una ritidectomía con buenos resultados hay que comprender la anatomía facial y se requiere una apreciación del sistema musculoaponeurótico superficial de la cara y de su singular diferencia con respecto a la fascia superficial en otras partes del cuerpo. Los músculos faciales superficiales (los músculos de la expresión facial) están englobados dentro de la fascia superficial y mueven el tejido blando facial sobre el esqueleto facial. Esto contrasta con los músculos esqueléticos en otras partes del cuerpo, incluidos los músculos de la masticación, que se encuentran por debajo de la fascia profunda. ${ }^{1}$

La importancia de la fascia superficial de la cara en el envejecimiento facial y su uso en el rejuvenecimiento de la región malar y el cuello fue demostrado por primera vez por Skoog en 1969. El hecho de que la laxitud de la piel facial podría corregirse utilizando esta fascia, sin aplicar tensión directamente a la piel, fue una revelación de profunda significación para la técnica de ritidectomías. ${ }^{2}$

La razón anatómica del éxito de la plicatura de la fascia se hizo más comprensible a partir del trabajo clásico de Mitz y Peyronie, quienes introdujeron el término «sistema musculoaponeurótico superficial» (SMAS) para describir la anatomía de la capa profunda de la fascia superficial. El SMAS explica la relación y las COnexiones entre la fascia superficial, los músculos de la expresión facial y la dermis suprayacente. La parte importante del SMAS en el envejecimiento facial es sobre la parte más expresiva del rostro donde se encuentran los músculos periorales y perioculares. En el espacio entre las fascias superficiales y profundas existe el plano sub-SMAS, que se conoce comúnmente como plano profundo. La anatomía de este plano es compleja y sólo ahora se comprende de una manera que es útil para el cirujano. En el plano sub-SMAS se presentan cuatro tipos de estructura: los anexos profundos de los músculos faciales, los ligamentos de retención de la cara, los espacios areolares y las ramas nerviosas faciales. ${ }^{3}$

Los componentes adjuntos entre el SMAS y las estructuras más profundas fueron reco- nocidos por Papillon ${ }^{4}$ y la liberación de estos componentes adjuntos - desde la parte inferior del SMAS al periostio sobre el cigoma - permitió un nuevo ajuste quirúrgico, mejorado del medio SMAS a los compartimentos adjuntos. Ulteriormente, los compartimentos y puntos de fijación fueron descritos por Furnas en 1989 y denominados como los ligamentos de retención de la mejilla. ${ }^{5}$ También se reconoció que los ligamentos de retención continúan en la dermis. Los ligamentos cutáneos maseteros cerca del borde anterior del masetero se describieron posteriormente. ${ }^{4}$

En este momento se aprecia que los ligamentos de retención de la cara tienen una disposición formal para la unión de la fascia superficial al esqueleto facial. Los ligamentos tienen un patrón general concéntrico alrededor de las cavidades orbitaria y oral. Los ligamentos alrededor de la cavidad oral tienen una disposición en forma de C, formada por el grupo transverso de ligamentos cigomáticos en el extremo lateral, del cual descienden los ligamentos cutáneos maseteros verticales dispuestos hasta el ligamento mandibular. ${ }^{5}$

En general, hay una línea vertical de inserción de los ligamentos faciales temporal, periorbitario y perioral a lo largo de la unión entre la cara anterior y la cara lateral. Esta línea coincide con la extensión lateral de la fijación esquelética de los músculos de la expresión facial para la cara anterior. ${ }^{6}$

La fijación de los ligamentos a la dermis explica la forma de los surcos cutáneos en la cara. Entre los surcos aparecen pliegues y protuberancias de la piel porque los tejidos blandos subyacentes a estos son más móviles y no están restringidos en su posición al retener los ligamentos; por lo tanto, los ligamentos son responsables de los surcos. ${ }^{7}$ El efecto de la atrición de los ligamentos con el envejecimiento es más evidente cuando los ligamentos son más finos, porque están en la periferia de su extremo externo. ${ }^{8}$

El grupo de estructuras que ocupa el área más grande en el intervalo entre las fascias superficiales y profundas son los espacios faciales. Estos fueron descritos originalmente como áreas de tejido conjuntivo areolar laxo, pero de hecho son verdaderos espacios similares a hendiduras dentro del tejido blando de la cara, 
con la inclusión de las cavidades esqueléticas, unidas forman los espacios de la cara.

Nuestro propósito es avanzar en la comprensión de la anatomía facial al señalar que existen verdaderos espacios en la cara que ofrecen ventajas para el cirujano en su experiencia quirúrgica. ${ }^{9}$ La palabra «espacio» sugiere que está presente un vacío de tejido blando. De forma similar a los espacios pleurales en el tórax, los espacios faciales aparecen «cerrados» debido a las capas de tejido límite (pleura parietal y visceral) que se formaron in situ. El verdadero espacio se revela sólo cuando se ingresa al espacio (tórax o hendidura facial) durante la cirugía. ${ }^{10}$

En el tercio inferior de la cara, el espacio premaestro cubre la parte inferior de la fascia maseterina y permite el movimiento de la mandíbula con relación suprayacente. La laxitud del techo y de los aditamentos en el límite anteroinferior del espacio premaestro da como resultado la laxitud del platisma que lo cubre, que después se abomba para formar la papada. Los cambios de los espacios ocurren con el proceso de envejecimiento, pero éstos no son uniformes. La cantidad de cambio se relaciona con la cantidad de movimiento que ocurre en el área del espacio particular, siendo el movimiento de la mandíbula el más grande y el movimiento del templo el menor. ${ }^{11}$

El cambio de envejecimiento consiste en una disminución de la rigidez del espacio, debido a la distensión del techo. Posteriormente, esta laxitud del techo aparece como un abultamiento superficial por montículos malares sobre la mitad de la mejilla y papada sobre la mandíbula. En general, hay una expansión asociada de las dimensiones del espacio secundario a la laxitud de la fijación ligamentosa en el límite del espacio y bolsas de párpados inferiores que se extienden sobre el borde orbitario inferior. Es más fácil diseccionar los espacios envejecidos y el tejido areolar dentro de las separaciones a medida que se abren. ${ }^{12}$

Los espacios son «seguros» quirúrgicamente porque son áreas que están completamente libres de anatomía importante. Todos los nervios, vasos, ligamentos y músculos están fuera de los espacios faciales. Los ligamentos de retención proporcionan el refuerzo que determina la ubicación específica de la membrana límite.
En algunas áreas, las ramas del nervio facial se dirigen directamente por fuera de la membrana de revestimiento. ${ }^{13}$

La técnica de disección utilizada cuando se opera en el plano sub-SMAS debe estar relacionada con el carácter del área particular que se diseca. Dentro de los espacios, la disección roma es todo lo que se requiere para extenderse rápidamente a los límites del espacio. A medida que la disección se abre, una hendidura preexistente en las capas de tejido no produce sangrado. Esto contrasta con la disección subcutánea habitual de la cara, que es sólo unos milímetros más superficial y está en la superficie del músculo que forma parte del techo del espacio, ya sea platisma u orbicular. En consecuencia, cuando la disección se realiza correctamente, se evitan los hematomas y no existe el riesgo de daño del nervio facial. Cuando se opera fuera de los espacios, la técnica de disección se cambia a disección roma precisa con el fin de separar ramas nerviosas faciales, si es necesario, lejos de ligamentos faciales, antes de lisar las bandas ligamentosas necesarias. ${ }^{7}$

El principio de corrección quirúrgica de los cambios de envejecimiento, cuando se opera en el plano sub-SMAS, implica avanzar el colgajo compuesto de tejido laxo en la dirección requerida y fijarlo ahí. Por lo general, el área de laxitud que requiere corrección se encuentra en la parte medial de uno de los espacios. La cantidad de liberación quirúrgica requerida en el límite medial del espacio está determinada por el estado de los ligamentos de retención en relación con el límite medial. ${ }^{14}$

La mayoría de técnicas de ritidoplastia implican incisiones periauriculares, manipulación y disección del SMAS y resección cutánea. Las primeras técnicas involucraban únicamente la resección de piel excedente sin mayor manejo de tejidos profundos. Mitz y Peyronie describieron el SMAS, y Skoog, en 1974, lo describe como una capa de tejido conectivo denso profundo al plano cutáneo. ${ }^{15}$

Ritidoplastia subcutánea: las técnicas de sólo piel datan de los primeros años de las ritidoplastias en las cuales se diseca de manera subcutánea y se tracciona en dirección de un vector. Tiene múltiples factores positivos y negativos. Es rápida y relativamente segura; sin embargo, mantiene las propiedades del enve- 
jecimiento de la piel y no durará tanto como otras técnicas profundas. Además, al aplicar toda la tensión de cierre en la piel, puede tener secuelas como distorsiones del trago, deformidad en el lóbulo de la oreja, como secuela de ritidectomía y alteración de la línea capilar. ${ }^{16}$

Ritidoplastia subcutánea con manipulación del SMAS: las técnicas «S-lift» de Fulton y «MACS lift» (minimal access cranial suspension) de Tonnard, basan su objetivo en levantar y suspender el cuello, surcos nasogenianos y tercio medio facial mediante suturas en el SMAS. Existen diferentes descripciones de vectores usados en las plicaturas del SMAS. Algunas complicaciones de estas técnicas son las irregularidades por tracción y lesión nerviosa por la sutura. Se han reportado tasas menores de complicaciones en comparación con otras técnicas más profundas. Esta es la técnica empleada en nuestra descripción, modificando los puntos de vectores de los mismos en tres puntos específicos para los diferentes tercios. ${ }^{17}$

SMASectomía lateral: Baker describió esta técnica en la que se reseca una tira del SMAS paralela al pliegue nasolabial sobre el borde anterior de la parótida, ${ }^{5}$ generalmente se marcan porciones del SMAS de 2 a $4 \mathrm{~cm}$ de ancho para resecar. Se menciona una serie de ventajas sobre las técnicas de elevación profunda del SMAS, incluyendo la integridad de la fascia superficial, menor riesgo de daño nervioso debido a que la mayoría de la disección es sobre la parótida y la fijación prolongada del SMAS que evita dehiscencias postoperatorias. ${ }^{18}$

Técnica supra-high y high SMAS: planificación del colgajo más alto o a lo largo del borde superior del arco cigomático y extiende la disección medialmente para movilizar el tejido de la cara media, supera la arruga del tercio medio y produce un resultado mejorado. Los beneficios de la técnica high SMAS incluyen una elevación combinada, equilibrada y armoniosa de los tejidos caídos de la cara media, la mejilla y la línea de la mandíbula, sin la necesidad de realizar un procedimiento de elevación de la cara media separado o distinto. Esta técnica descrita por Timothy Marten fue mejorada en 2002; no obstante, una variante modificada que realizamos en esta descripción es hacer una disección arriba del arco cigomático en la fosa temporal, elevando el tejido subcutáneo de la patilla para evitar el desplazamiento de la misma. Se realiza disección del músculo temporal superficial hasta identificar la fascia temporal profunda, donde se fijará el vector temporal del colgajo del SMAS. Esta técnica dará un soporte en el tercio medio y región periorbitaria de la ritidectomía y el segundo colgajo del SMAS se llevará como vector del tercio inferior, fijándolo en la mastoides. ${ }^{19}$

Existen muchas técnicas de ritidectomía y no hay evidencia suficiente para considerar que una sea superior a otra. La finalidad del

Tabla 1: Descripción de la muestra en estudio.

\begin{tabular}{lc} 
& $\mathrm{n}(\%)$ \\
\hline Tamaño muestral & $39(100.0)$ \\
Sexo & \\
$\quad$ Femenino & $33(84.6)$ \\
Masculino & $6(15.4)$ \\
Procedimiento & \\
Plicatura de SMAS & $19(48.7)$ \\
Supra-high SMAS & $20(51.3)$ \\
Tiempo quirúrgico (horas) & \\
1-3 & $19(48.7)$ \\
3-6 & $20(51.3)$ \\
Ritidosis periocular & \\
Presente & $30(76.9)$ \\
Ausente & $9(23.1)$ \\
Corrección periocular & \\
Realizada & $33(84.6)$ \\
No realizada & $6(15.4)$ \\
Ritidosis tercio facial medio & \\
Presente & $39(100.0)$ \\
Ausente & $0(0.0)$ \\
Corrección tercio facial medio & \\
Realizada & $39(100.0)$ \\
No realizada & $0(0.0)$ \\
Ritidosis tercio facial inferior & \\
Presente & \\
Ausente & \\
Aorrección tercio facial inferior & \\
Presente & \\
Ausente & \\
1105-1050 & \\
\hline
\end{tabular}

SMAS = Sistema musculoaponeurótico superficial. 
presente trabajo es comparar dos técnicas de ritidectomía: supra-high SMAS (SH-SMAS) y plicatura SMAS (P-SMAS), para demostrar con cuál se obtienen los mejores resultados con menos complicaciones, según nuestra experiencia y muestra.

\section{MATERIAL Y MÉTODO}

Se trata de un estudio longitudinal, comparativo, observacional, retrospectivo, efectuado en el Servicio de Cirugía Plástica y Reconstructiva del American British Cowdray Medical Center con los expedientes clínicos y registros del seguimiento de pacientes que referían inconformidad con su aspecto estético facial, identificados como caucásicos, con escala de Fitzpatrick 1-4 en su mayoría, en total 39 pacientes: 20 operados con la técnica SH-SMAS y 19 con la técnica P-SMAS, seis mujeres (15.4\%) y 33 hombres (84.6\%); todos los pacientes tenían ritidosis del tercio facial medio, 30 (76.9\%) ritidosis periocular y $34(87.2 \%)$ ritidosis del tercio facial inferior. Veintinueve pacientes (74.4\%) con un ángulo cervicofacial de $105-90^{\circ}$ y 10 (25.6\%) de $110-105^{\circ}$.
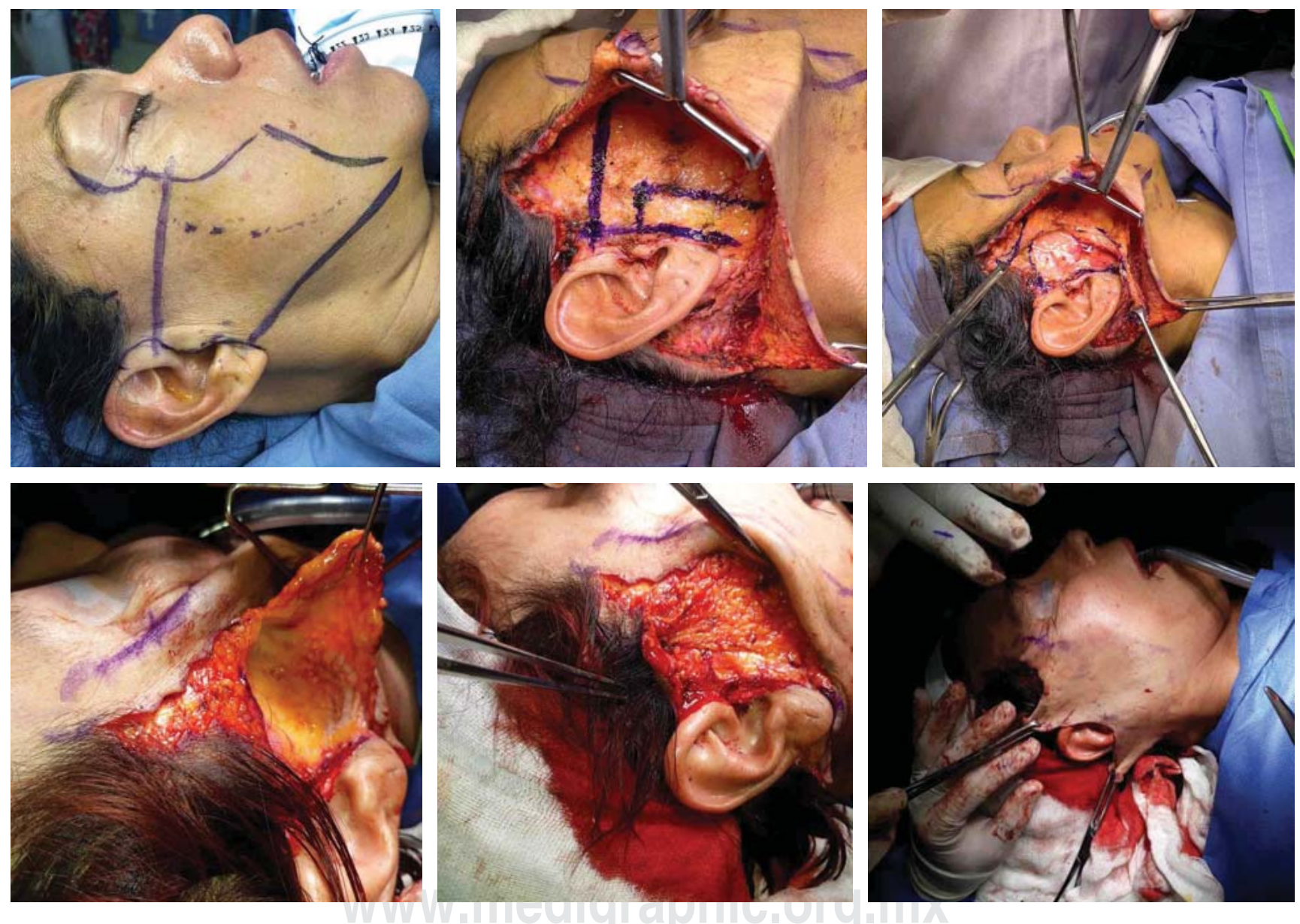

Figura 1: Marcaje quirúrgico de la técnica supra-high SMAS con delimitación de disección subcutánea y del SMAS. Marcaje quirúrgico de colgajo en «7》 invertido para dos vectores. Vector temporal y vector mastoideo. Disección de colgajo del SMAS hasta identificar la fascia parotídeo maseterina. Colgajos del SMAS en dos vectores. Postoperatorio inmediato de ritidectomía supra-high SMAS. Disección subcutánea. Se identifica SMAS para iniciar con colgajos. Disección del SMAS hasta fascia parotídea maseterina. Colgajo del SMAS en vector temporal. Se eleva plano de patilla y se diseca hasta identificar la fascia temporal profunda. Se fija el colgajo. Colgajo del SMAS en vector temporal fijado a la fascia temporal profunda. Compensación de colgajos cutáneos.

SMAS = Sistema musculoaponeurótico superficial. 

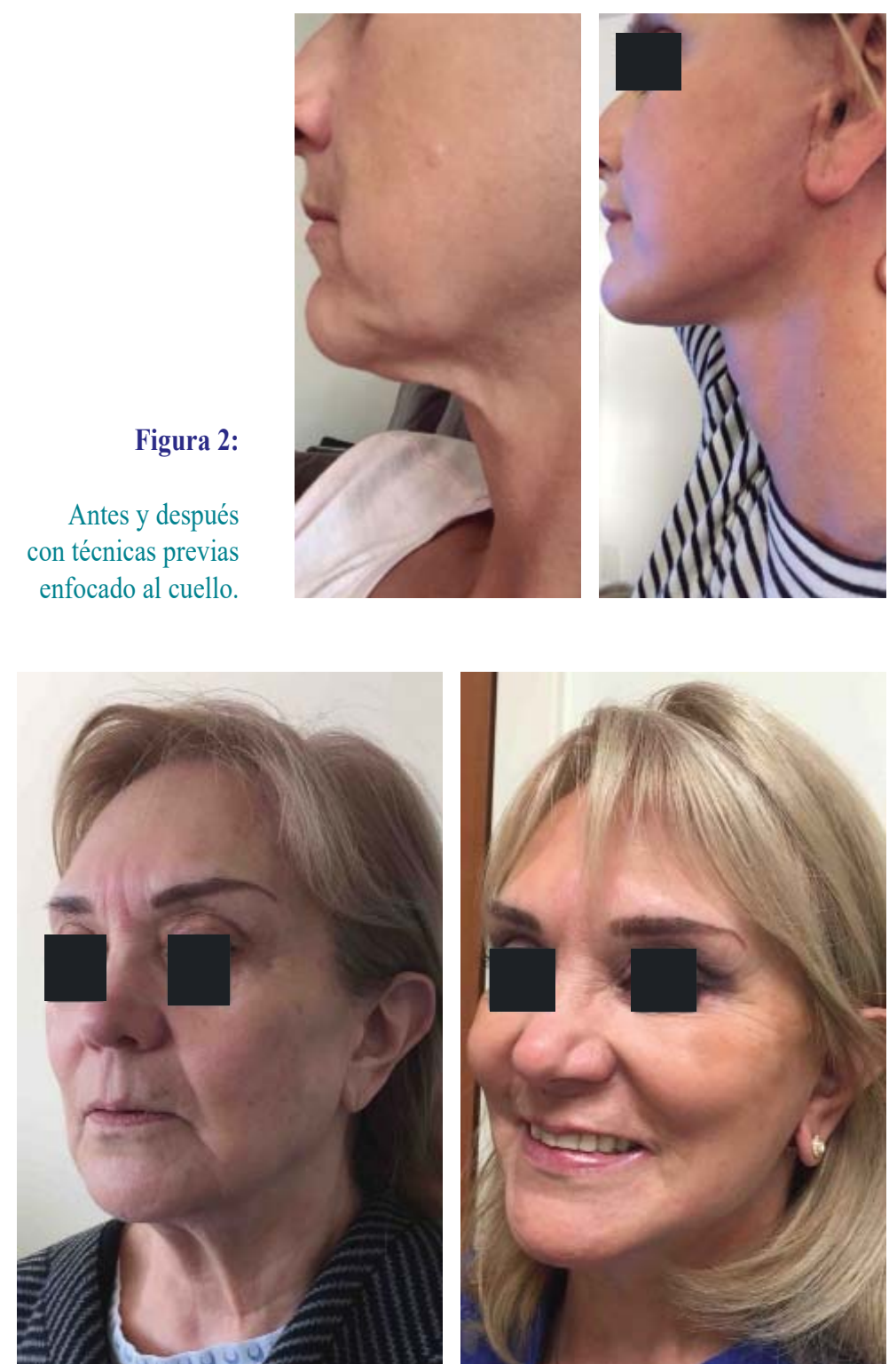

Figura 3: Antes y después con técnica supra-high SMAS.

SMAS = Sistema musculoaponeurótico superficial.

Los pacientes seleccionados cursaban con algún grado de rítides en tercio medio e inferior facial, líneas de marioneta, código de barras, disminución del ángulo cervicofacial, pérdida de la posición natural del complejo muscular de la región malar, o pérdida de la altura y función del músculo depresor del ángulo del labio, así como múltiples rítides superficiales y profundas al mismo nivel. Se sometieron a evaluación clínica y se identificaron los casos

quirúrgicos para el procedimiento de ritidectomía, se analizó el ángulo cervicofacial y la debilidad del SMAS en diferentes puntos. Todos los pacientes aceptaron el procedimiento quirúrgico al que correspondía el grupo según sus características, firmaron el consentimiento informado sobre el estudio y el procedimiento quirúrgico. Los pacientes estaban conscientes del uso de fotografías para uso académico.

Anteriormente se realizó el procedimiento bajo anestesia general utilizando la técnica $\mathrm{SH}$ SMAS, y posteriormente, ante la facilidad y la comodidad de la técnica con P-SMAS, optamos por esta última con aquéllos que se presentaran a partir de ese momento, por lo cual no fue aleatorizado. Lo analizamos como comparación entre 19 casos de (P-SMAS) y 20 controles (SH-SMAS) que se operaron en distinto momento. No se realizó ningún procedimiento bajo conveniencia, sino de acuerdo con la planificación previa de los pacientes que acudieron a valoración para ritidectomía y se asignó el plan quirúrgico en la misma valoración por el mismo equipo quirúrgico. Asimismo, todos los casos fueron intervenidos por el mismo equipo quirúrgico (Tabla 1).

Los datos se recabaron con base en el expediente clínico y la información registrada en las consultas de seguimiento de cada uno de los pacientes de ambos grupos: al mes, a los tres meses y a los 12 meses del postoperatorio.

\section{Técnica supra-high SMAS}

Marcaje quirúrgico con delimitación de la disección subcutánea y del SMAS con marcaje del colgajo en «7》 invertido para dos vectores, disección del colgajo del SMAS hasta identificar la fascia parotídeo maseterina y colgajo en su vector temporal. Se eleva el plano de la patilla y se diseca hasta identificar la fascia temporal profunda y se fija el colgajo a dicha fascia y se compensan los colgajos cutáneos (Figura 1).

\section{Técnica subcutánea con plicatura en tres vectores}

Marcaje quirúrgico, disección máxima en plano subcutáneo con prueba para identificar los dos vectores de tercio medio con pinzamiento del SMAS. Marcaje de SMASectomía para vectores de tercio medio y SMASectomía en bumerán 
con desplazamiento de dos vectores en tercio medio. Primera sutura del vector principal para plicatura del SMAS, disección del tercer vector en tercio inferior con colgajo del SMAS a nivel de platisma, disección profunda del SMAS en tercio inferior con límite subplatisma en la línea media. Colgajo del SMAS para el tercer vector y su respectiva plicatura (Figura 2).
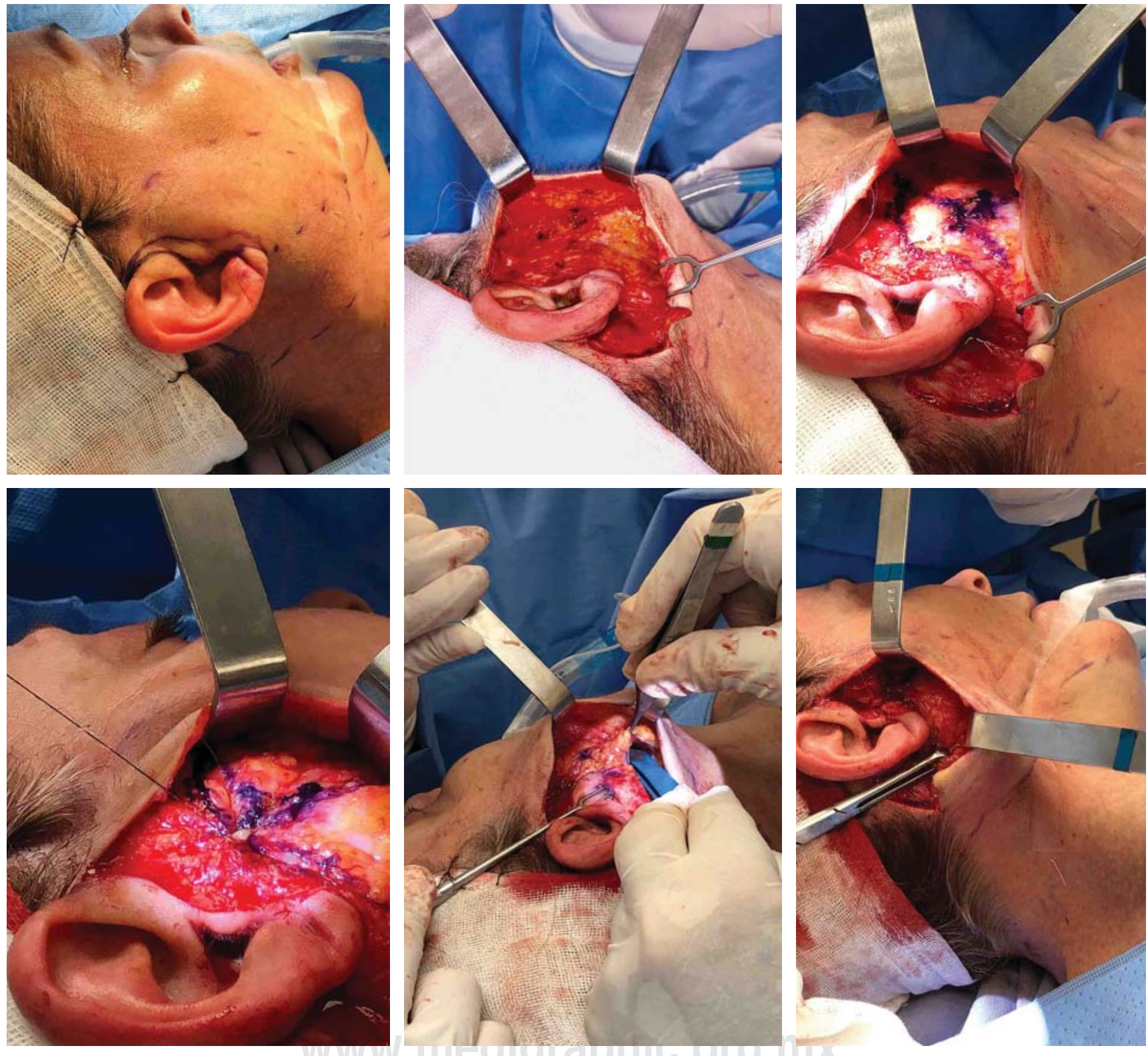

Figura 4: Paciente con rítides grado 4 en tercio medio e inferior, candidata para técnica subcutánea con plicatura en tres vectores. Marcaje quirúrgico de técnica con plicatura en tres vectores. Disección máxima en plano subcutáneo. Prueba para identificar los dos vectores de tercio medio con pinzamiento del SMAS. Marcaje de SMASectomía para vectores de tercio medio. SMASectomía en bumerán con desplazamiento de dos vectores en tercio medio. Primera sutura de vector principal para plicatura del SMAS. Disección de tercer vector en tercio inferior con colgajo del SMAS a nivel de platisma. Disección profunda del SMAS en tercio inferior con límite subplatisma a línea media. Colgajo del SMAS para tercer vector y su respectiva plicatura.

SMAS = Sistema musculoaponeurótico superficial. 

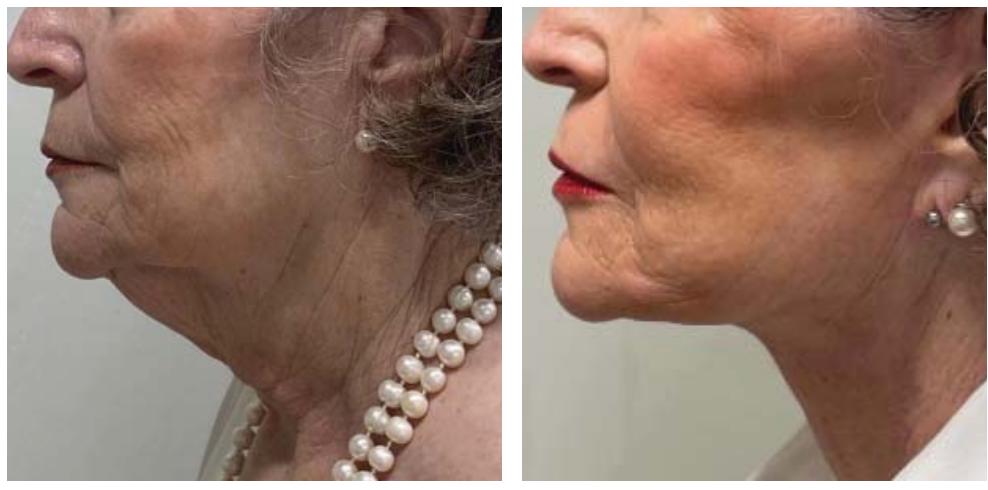

Figura 5: Comparación antes y después.

Tabla 2: Descripción de la muestra en estudio.

\begin{tabular}{lc} 
& $\mathrm{n}(\%)$ \\
\hline $\begin{array}{l}\text { Porcentaje de satisfacción } \\
\text { postoperatoria al final del }\end{array}$ & \\
seguimiento & \\
$80-100$ & $29(74.4)$ \\
$60-79$ & $5(12.8)$ \\
$<60$ & $5(12.8)$ \\
Total & $39(100.0)$ \\
Complicaciones postoperatorias & \\
Ninguna & $33(84.6)$ \\
Hematoma & $3(7.7)$ \\
Asimetría facial & $2(5.1)$ \\
Lesión nerviosa & $1(2.6)$ \\
Total & $39(100.0)$ \\
\hline
\end{tabular}

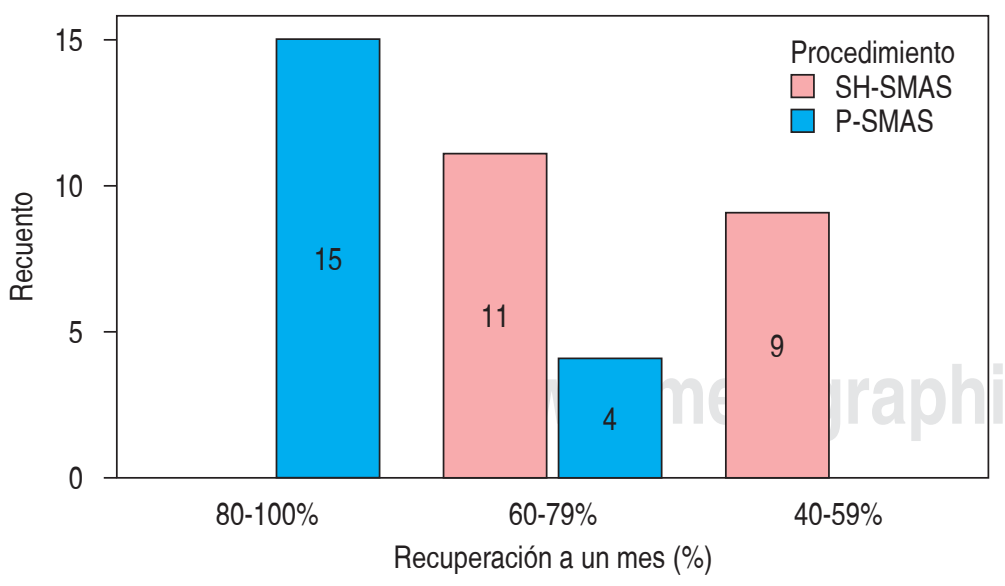

Figura 6: Recuperación a un mes según la técnica de ritidectomía utilizada. SMAS = Sistema musculoaponeurótico superficial. SH-SMAS = supra-high SMAS. P-SMAS = plicatura SMAS.

\section{RESULTADOS}

De los 39 pacientes, cinco quedaron con una satisfacción inferior a $60 \%$, otros cinco con satisfacción de 60 a 79\% y 29 (74.4\%) con satisfacción igual o superior a $80 \%$. Cabe mencionar que no hubo diferencias estadísticamente significativas entre la técnica utilizada y la probabilidad de presentar una satisfacción estética $\geq 80 \%, \mathrm{OR}=$ 0.93 (IC 95\%, 0.22-1.42) (Figuras 2 a 5).

En cuanto a complicaciones, un caso $(2.56 \%)$ tuvo lesión nerviosa en el grupo de pacientes operados con SH-SMAS, dos (5.12\%) asimetría facial, tres (7.39\%) hematoma y 33 $(84.6 \%)$ sin complicaciones. No hubo diferencias estadísticamente significativas entre la técnica utilizada y la probabilidad de no presentar complicaciones postoperatorias, $\mathrm{OR}=2.13$ (IC 95\%, 0.34-13.24) (Tabla 2).

En cuanto a duración del procedimiento quirúrgico, todos los pacientes operados con SH-SMAS tuvieron una duración quirúrgica igual o mayor a tres horas y los operados con P-SMAS menor a tres horas (Figura 6).

En cuanto a la recuperación, en el primer mes $78.9 \%$ de los pacientes operados con P-SMAS tuvieron una recuperación suficiente $(\geq 80 \%)$, mientras que ninguno de los pacientes operados por SH-SMAS alcanzó el 80\%, sino hasta el tercer mes de seguimiento. Por medio de un análisis de supervivencia se proyecta que los pacientes de P-SMAS se recuperaron lo suficientemente en 1.421 meses (IC 95\%, 1.044-1.798), mientras que los pacientes operados con SH-SMAS lo hicieron en 7.050 meses (IC 95\%, 5.037-9.063); $\chi^{2}$ de 26.554 por Log Rank de Mantel-Cox, p a dos colas $<0.000$ (Tablas 3 y 4) (Figuras 7 y 8).

\section{DISCUSIÓN}

Las cirugías con fines estéticos, incluida la operación de ritidectomía, se están volviendo cada vez más populares y el rejuvenecimiento sigue siendo uno de los procedimientos estéticos más solicitados. Los procedimientos se pueden utilizar para reducir la flacidez de la piel y los tejidos subcutáneos y crear un rostro más juvenil reestructurando la anatomía de la cara. La ritidectomía se considera generalmente del tercio medio e inferior, ya que la ritidectomía coronal o del tercio superior ha ido perdiendo preferencia, 
ya que los pacientes en la actualidad enfatizan en la naturalidad y en menos cicatrices.

Las técnicas de ritidectomía clásicas se pueden dividir por planos, sea subcutáneo, con plicatura del SMAS, mastectomía y ritidectomía con colgajos del SMAS y tejidos profundos. Las complicaciones asociadas con los procedimientos de la ritidectomía son raras, pero clínicamente importantes como hematomas, necrosis cutánea de los bordes de la herida, infección, lesiones nerviosas, alopecia y los estigmas de la ritidectomía. Hoy en día, la tendencia es hacer procedimientos mínimamente

Tabla 3: Resultados principales: recuperación postoperatoria según el tiempo de seguimiento.

\begin{tabular}{cccccc} 
& \multicolumn{3}{c}{$\%$ de recuperación } & & Significación aproximada \\
\cline { 2 - 5 } Meses de seguimiento & $\begin{array}{c}80-100 \\
\mathrm{n}(\%)\end{array}$ & $\begin{array}{c}60-79 \\
\mathrm{n}(\%)\end{array}$ & $\begin{array}{c}40-59 \\
\mathrm{n}(\%)\end{array}$ & Total & $\chi^{2}$ (Log Rank de Mantel-Cox) \\
\hline 1 & $15(38.5)$ & $15(38.5)$ & $9(23.1)$ & $39(100.0)$ & $0.000^{*}$ \\
3 & $30(76.9)$ & $9(23.1)$ & $0(0.0)$ & $39(100.0)$ & $39(100.0)$ \\
\hline
\end{tabular}

* La significación aproximada o valor de $\mathrm{p}<0.05$ orienta a rechazar la hipótesis nula. Ver el apartado de resultados.

Tabla 4: Porcentaje de satisfacción postoperatoria estratificado por las siguientes dos variables: complicaciones postoperatorias y técnica de ritidectomía realizada.

\begin{tabular}{|c|c|c|c|c|c|c|c|}
\hline \multirow[b]{2}{*}{$\begin{array}{l}\text { Complicaciones } \\
\text { postoperatorias }\end{array}$} & \multirow[b]{2}{*}{$\begin{array}{l}\text { Técnica de } \\
\text { ritidectomía }\end{array}$} & \multicolumn{3}{|c|}{ Satisfacción postoperatoria } & \multirow[b]{2}{*}{$\begin{array}{c}\text { Total } \\
\text { (n) }\end{array}$} & \multicolumn{2}{|c|}{ Significación aproximada } \\
\hline & & $80-100 \%$ & $60-79 \%$ & $<60 \%$ & & $\chi^{2}$ de Pearson & $\begin{array}{l}\text { Coeficiente de } \\
\text { contingencia }\end{array}$ \\
\hline \multirow[t]{3}{*}{ Ninguna } & Supra-high SMAS & 15 & 1 & 0 & 16 & $0.512 *$ & $0.512 *$ \\
\hline & Plicatura de SMAS & 14 & 2 & 1 & 17 & & \\
\hline & Total & 29 & 3 & 1 & 33 & & \\
\hline \multirow[t]{3}{*}{ Hematoma } & Supra-high SMAS & & 2 & 0 & 2 & $0.083^{*}$ & $0.083^{*}$ \\
\hline & Plicatura de SMAS & & & 1 & 1 & & \\
\hline & Total & 0 & 2 & 1 & 3 & & \\
\hline \multirow[t]{3}{*}{ Asimetría facial } & Supra-high SMAS & & & 1 & 1 & No calculable & No calculable \\
\hline & Plicatura de SMAS & & & 1 & 1 & & \\
\hline & Total & 0 & 0 & 2 & 2 & & \\
\hline \multirow[t]{3}{*}{ Lesión nerviosa } & Supra-high SMAS & & & 1 & 1 & No calculable & No calculable \\
\hline & Plicatura de SMAS & & & 0 & 0 & & \\
\hline & Total & 0 & 0 & 1 & 1 & & \\
\hline Supra-high SMAS & & 15 & 3 & 2 & 20 & $0.815^{*}$ & $0.815^{*}$ \\
\hline Plicatura de SMAS & & 14 & 2 & 3 & 19 & & \\
\hline Total (n) & & 29 & 5 & 5 & 39 & & \\
\hline
\end{tabular}




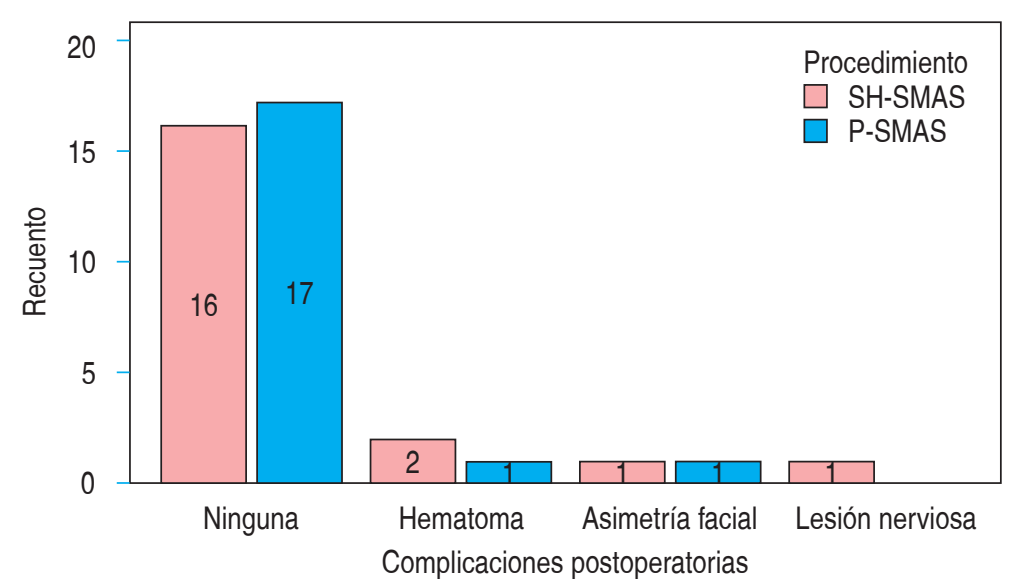

Figura 7: Complicaciones postoperatorias según la técnica de ritidectomía utilizada. SMAS = Sistema musculoaponeurótico superficial. SH-SMAS = supra-high SMAS. P-SMAS = plicatura SMAS.

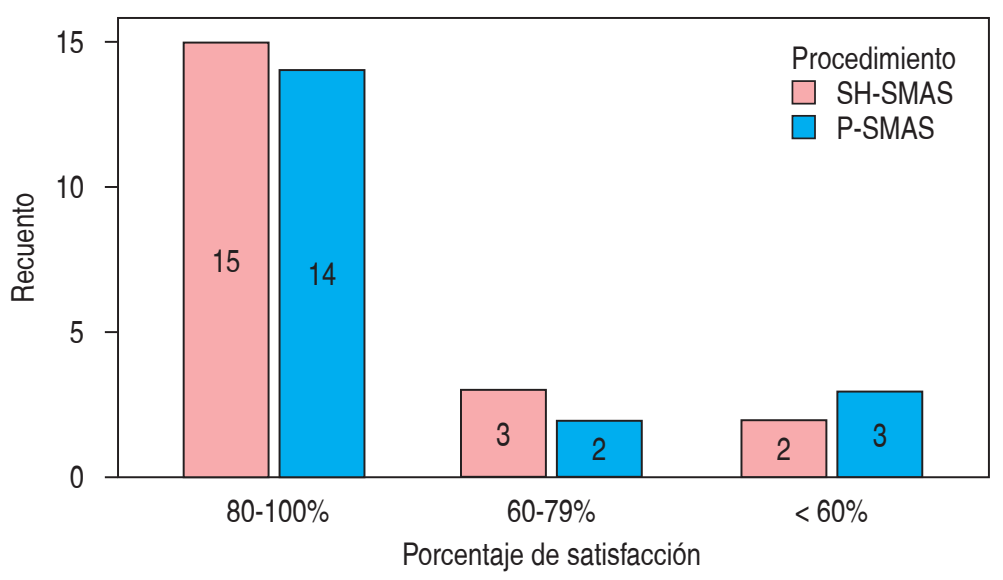

Figura 8: Porcentaje de satisfacción estética según la técnica de ritidectomía utilizada. SMAS = Sistema musculoaponeurótico superficial. SH-SMAS = supra-high SMAS P-SMAS = plicatura SMAS.

invasivos con menor riesgo de complicaciones, con un periodo de recuperación más corto. ${ }^{20}$

El envejecimiento es causado por muchos factores: la exposición excesiva al sol, fumar o beber pueden envejecer significativamente a una persona, así como la genética, los factores ambientales y el lugar de vivienda. Las investigaciones sugieren que fumar durante 10 años envejece a una persona entre dos y cinco años. Al realizar algún tipo de ritidectomía es importante determinar el plano en el que se realizará el procedimiento. Uno de los espacios virtuales más determinantes en la ritidectomía es el es- pacio precigomático, mismo que es superior al cuerpo del cigoma; en esta zona es donde la plicatura tiene su primer vector, así como en la fosa temporal. En el borde superior son los ligamentos de retención del párpado y la mejilla, el borde inferior cubre los orígenes superiores del cigomático mayor, menor y el músculo elevador del labio superior, esto determina el segundo vector en las ritidectomías con el SMAS, y su respectivo colgajo o plicatura es la ritidectomía del tercio medio. Las porciones laterales alcanzan el borde posterior del cigoma y no hay nervios faciales que atraviesen este espacio, solo el nervio cigomático facial para disecar a través del espacio sub-SMAS en la mejilla inferior en el espacio, donde se describen las técnicas profundas de la fascia premaseterina. ${ }^{21}$

El borde inferior no tiene soporte ligamentoso y se encuentra aproximadamente $15 \mathrm{~mm}$ por encima del ángulo de la mandíbula. El borde superior es el borde superior del platisma; al extender la herida retroauricular se determina la cervicoplastia, dependiendo de si se realizan vectores retroauriculares con colgajo de platisma o no. El espacio entre el espacio precigomático y premasetero se conoce como el espacio premasetero medio, por debajo del piso de este espacio corren el conducto parotídeo y los troncos bucales superior e inferior. Algunas ramas de la rama marginal mandibular pasan sobre el espacio premasetero. El nervio pasa sobre el músculo masetero anteriormente $8 \mathrm{~mm}$ por encima del ángulo de la mandíbula; una rama corre por debajo de este piso del espacio y otra rama sobre este espacio, hasta el músculo platisma inferior. Al realizar una incisión preauricular del SMAS para disecar la capa de éste existe el riesgo de dañar la rama mandibular; esto se puede prevenir al colocar la incisión $30 \mathrm{~mm}$ por delante de la oreja.

La grasa submentoniana es diferente de la grasa bucal y está compuesta por dos compartimentos subcutáneos en el borde de la mandíbula y son los compartimentos superior e inferior de la papada. La grasa de la papada está separada de la grasa submandibular por el tabique mandibular, éste se interdigita con fibras del músculo platisma para unirse al borde anterior de la mandíbula, fijando la piel al borde de la misma. Esta es la razón por la que la piel del borde de la mandíbula es móvil 
en la dirección superior, pero no tanto en la dirección inferior. ${ }^{21}$

Furnas informó en 1989 sobre las adherencias densas (ligamento mandibular) localizadas en el tercio anterior del borde mandibular, pero no mencionó la adherencia presente en el tercio medio. Sólo unos pocos investigadores informaron que la papada se debe a cambios secundarios causados por la pérdida de volumen y Yousif indicó que son causados por la caída del tejido blando debido a las fuerzas gravitacionales y la laxitud del tejido.

Se cree que el tamaño normal del lóbulo de la oreja es de $20 \mathrm{~mm}$ y si es más grande puede verse como un signo de envejecimiento. ${ }^{22}$ McKinney informó que la longitud promedio del lóbulo de la oreja y la oreja era de $18 \mathrm{~mm}$. La relación entre la longitud del lóbulo de la oreja y la de la oreja es 28 y ésta aumenta con el envejecimiento. El rango normal de esta relación es de 25 a 30 y si excede de 33 se recomienda una reducción del lóbulo. Se puede obtener una apariencia más natural si la capa dérmica en la base del colgajo tragal se fija a la fascia o al cartílago subyacente, con dos a tres suturas de anclaje con prolene 5-0, para crear una depresión en la porción pretragal. ${ }^{23}$
Si bien se realiza cirugía plástica estética general para cambiar el aspecto innato de una persona, se realizan cirugías de rejuvenecimiento para solucionar los problemas causados por el envejecimiento, devolviendo al paciente a un estado más joven. Los problemas que aparecen en el rostro debido al envejecimiento incluyen arrugas, imperfecciones, manchas de la edad, pérdida de volumen y flacidez de tejidos. Cuando se ven de cerca, las arrugas son la principal causa de parecer viejo, mientras que desde lejos, la pérdida de volumen y la caída de los tejidos son las principales causas. Las arrugas comienzan a formarse a finales de la tercera década de la vida en lugares con la piel más delgada como el área periorbitaria y a medida que las personas envejecen aumenta la pérdida de volumen y la caída de tejidos. Estos problemas no se pueden afrontar con un solo procedimiento. $^{24}$

Para abordar el problema de las arrugas o la pérdida de volumen, procedimientos relativamente simples como la toxina botulínica, rellenos e inyecciones de grasa pueden ser suficientes, pero para rejuvenecer una cara flácida, se realizan estiramientos faciales, métodos quirúrgicos no invasivos o mínimamente invasivos.
Figura 9:

Comparación de las respectivas recuperaciones de

las dos técnicas de ritidectomía: plicatura de SMAS

(línea verde) y supra-high SMAS (línea roja)

SMAS $=$ Sistema musculoaponeurótico superficial.

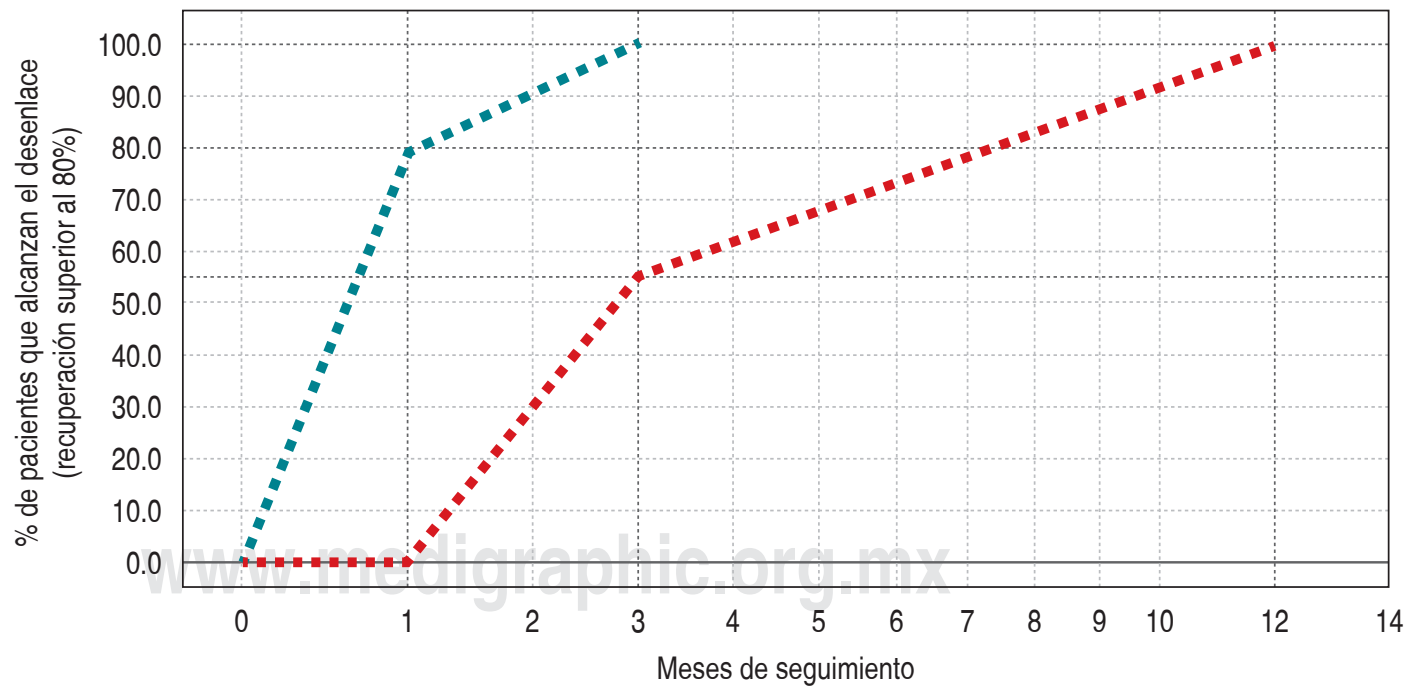

Se presentan dos curvas de Kaplan-Meier en función de uno menos la supervivencia, en las cuales el factor de desenlace es la recuperación postoperatoria igual o superior a $80 \%$. 1) Al primer mes de seguimiento $80 \%$ de los pacientes con plicatura de SMAS (línea verde) alcanzaron el desenlace. 2) A los tres meses de seguimiento, 100\% de los pacientes con plicatura de SMAS alcanzaron el desenlace, contra 55\% de los pacientes con supra-high SMAS (línea roja). 3) A los 12 meses de seguimiento, 100\% de los pacientes con supra-high SMAS alcanzaron el desenlace. 
Tabla 5. Probabilidad de satisfacción estética final $\geq 80 \%$.

Satisfacción estética Satisfacción estética final $\geq 80 \% \quad$ final $<80 \%$

\begin{tabular}{lrrr}
\hline Plicatura-SMAS & 14 & 5 & 19 \\
Supra Hight-SMAS & 15 & 5 & 20 \\
& 29 & 10 & 39 \\
\hline
\end{tabular}

$\mathrm{OR}=0.93$ (IC 95\%, 0.22-1.42). No hay diferencia estadísticamente significativa entre ambas técnicas y la probabilidad de presentar una satisfacción estética final $\geq 80 \%$. SMAS = Sistema musculoaponeurótico superficial.

\section{Tabla 6. Probabilidad de no presentar complicaciones postoperatorias.}

\begin{tabular}{lccc} 
& $\begin{array}{c}\text { Ausencia de } \\
\text { complicaciones } \\
\text { postoperatorias }\end{array}$ & $\begin{array}{l}\text { Presencia de 1 } \geq \\
\text { complicaciones } \\
\text { postoperatorias }\end{array}$ & \\
\hline Plicatura-SMAS & 17 & 2 & 19 \\
Supra Hight-SMAS & 16 & 4 & 20 \\
& 33 & 6 & 39 \\
\hline
\end{tabular}

$\mathrm{OR}=2.13$ (IC 95\%, 0.34-13.24). No hay diferencia estadísticamente significativa entre ambas técnicas y la probabilidad de no presentar complicaciones postoperatorias. SMAS $=$ Sistema musculoaponeurótico superficial.

\section{Tabla 7. Recuperación al primer mes $\geq 80 \%$.}

\begin{tabular}{lccc} 
& $\begin{array}{c}\text { Recuperación al } \\
\text { primer mes } \geq 80 \%\end{array}$ & $\begin{array}{c}\text { Recuperación al } \\
\text { primer mes }<80 \%\end{array}$ & \\
\hline Plicatura-SMAS & 15 & 4 & 19 \\
Supra Hight-SMAS & $0 *$ & 20 & 20 \\
& 15 & 24 & 39 \\
\hline * OR = infinito. SMAS = Sistema musculoaponeurótico superficial.
\end{tabular}

Los estiramientos faciales pueden tener un periodo de recuperación más corto, pero los efectos son sólo temporales. A pesar de los periodos de recuperación ligeramente más largos, se deben realizar estiramientos faciales convencionales para obtener buenos resultados, maximizar la eficiencia de la cirugía y mantener los efectos duraderos. ${ }^{24}$
Durante el periodo de oro de la cirugía plástica, se describieron múltiples procedimientos y técnicas para ritidectomías que fueron pioneros de las respectivas técnicas. Se describieron accesos y técnicas más agresivas, prometiendo resultados más estéticos; sin embargo, las últimas técnicas se describen como de mínima invasión complementadas con variantes de las mismas, dando un mejor resultado en cuanto a lesiones y complicaciones, tiempo quirúrgico, disminución de estancia hospitalaria y mejoría en la recuperación. ${ }^{24}$

La homogenización de los datos se analiza con las prueba $\chi^{2}$ y Fisher, según corresponde con el tamaño de la muestra. Posteriormente, y respetando el diseño del estudio, utilizamos la razón de momios (OR, IC 95\%) como medida de asociación para aproximar la probabilidad de presentar los siguientes desenlaces, según la técnica utilizada (P-SMAS o SH-SMAS) (Figura 9, Tablas 5 y 6 ).

El cociente de las siguientes razones de momios (recuperación y duración del transoperatorio) tienden a infinito debido a que el denominador en la división es 0 (p. ej: 0 pacientes de SH-SMAS con recuperación superior al $80 \%$ al mes, o cero pacientes con SH-SMAS con transoperatorio menor a los tres meses) (Tablas 7 a 10).

Dado que se realizaron tres mediciones sobre recuperación (al primer mes, a los tres y 12 meses) fue posible realizar dos curvas de Kaplan-Meier para comparar qué tan rápido se recuperan los pacientes en ambas técnicas. El desenlace a comparar, es decir, la meta de recuperación suficiente que proponemos, es un porcentaje igual o superior a $80 \%$ según lo refiere el paciente. Es posible interpretar y comparar estas curvas restando el área debajo de la curva entre ambas y su posterior análisis de significancia estadística, utilizando la prueba Log Rank de Mantel-Cox, con la cual se obtuvo un valor $\chi^{2}$ de 26.554 , con valor de $\mathrm{p} \mathrm{a}$ dos colas $<0.000$. Limitadas al tiempo de recuperación más largo, se estiman medias y medianas para el tiempo de recuperación suficiente:

- Supra-high SMAS: 7.050 (5.037-9.063, IC 95\%). Dicho de otro modo, se proyecta que los pacientes operados con SH-SMAS se recuperan en aproximadamente siete meses. - Plicatura de SMAS: 1.421 (1.044-1.798, IC 95\%). Dicho de otro modo, se proyecta 
que los pacientes operados con P-SMAS se recuperan al mes y medio.

\section{CONCLUSIONES}

Una apreciación de los espacios de partes blandas de la cara descritos recientemente ofrece ventajas para el cirujano involucrado en el rejuvenecimiento facial. Los espacios deben buscarse y luego utilizarse, ya que son hendiduras preexistentes en los planos de los tejidos. Esto reduce la necesidad de gran parte de la disección quirúrgica tradicional, que se

\section{Tabla 8. Recuperación a los tres meses $\geq \mathbf{8 0} \%$.}

Recuperación a los Recuperación a los tres meses $\geq 80 \% \quad$ tres meses $<80 \%$

\begin{tabular}{llll} 
Plicatura-SMAS & $0^{*}$ & 19 & 19 \\
Supra Hight-SMAS & 9 & 11 & 20 \\
& 9 & 30 & 39 \\
\hline
\end{tabular}

* OR = infinito. SMAS = Sistema musculoaponeurótico superficial.

Tabla 9. Recuperación a los 12 meses $\geq 80 \%$.

Recuperación a los Recuperación a los 12 meses $\geq 80 \% \quad 12$ meses $<80 \%$

$\begin{array}{llll}\text { Plicatura-SMAS } & 0^{*} & 19 & 19 \\ \text { Supra Hight-SMAS } & 9 & 11 & 20 \\ & 9 & 30 & 39\end{array}$

* OR = infinito. SMAS = Sistema musculoaponeurótico superficial.

Tabla 10. Duración del transoperatorio $<3$ horas.

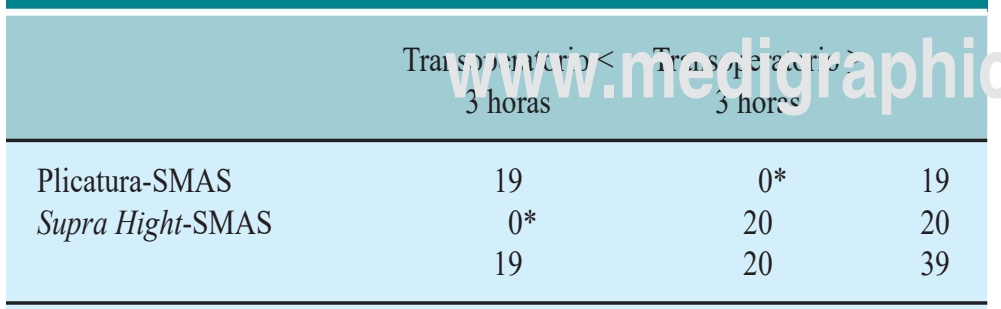

* OR = infinito. SMAS = Sistema musculoaponeurótico superficial. asocia con sangrado, hematomas y edema. Las ramas del nervio facial siempre están fuera de los espacios. Si se requiere una liberación quirúrgica adicional, es sólo en las áreas más pequeñas fuera de los espacios donde puede ser necesaria una liberación precisa de restricción ligamentosa residual.

Al poder analizar los resultados de las variables en nuestro control de casos con las dos técnicas quirúrgicas, podemos determinar -independientemente de la técnica- el tiempo quirúrgico y la amplia disección del complejo SMAS, que al realizar una adecuada técnica de plicatura en tres vectores del SMAS, los resultados estéticos son similares a corto y largo plazo con respecto a la técnica de ritidectomía con doble colgajo del SMAS descrita previamente, por lo que con una adecuada selección del paciente no es necesario someterlo a más de cuatro horas de tiempo quirúrgico y realizar disección amplia del SMAS, ya que existe mayor susceptibilidad de tener complicaciones como sangrado y lesión nerviosa. Realizando un procedimiento simple, completo y con la técnica adecuada, la ritidectomía con P-SMAS de tres vectores es nuestra mejor opción de acuerdo con nuestra experiencia quirúrgica.

\section{REFERENCIAS}

1. Skoog T. Plastic surgery: new methods and refinements. Philadelphia: Saunders 1974, p. 16.

2. Stuzin JM, Baker TJ, Gordon HL. The relationship of the superficial and deep facial fascia: relevance to rhytidectomy and aging. Plast Reconstr Surg 1992; 89: 441.

3. Stuzin JM. MOC-PSSM CME article: Face lifting. Plast Reconstr Surg 2008; 121 (Suppl): 1-19.

4. Tonnard P, Verpaele A, Monstrey S et al. Minimal access cranial suspension lift: a modified S-lift. Plast Reconstr Surg 2002; 109: 2074-2086.

5. Stuzin JM. Restoring facial shape in face lifting: The role of skeletal support in facial analysis and midface soft-tissue repositioning. Plast Reconstr Surg 2007; 119: 362-376; discussion 377-378.

6. Rohrich RJ, Ghavami A, Constantine FC, Unger J, Mojallal A. Lift-and-fill face lift: Integrating the fat compartments. Plast Reconstr Surg 2014; 133: 756e-767e.

7. Pitanguy I, Machado BH. Facial rejuvenation surgery: A retrospective study of 8788 cases. Aesthet Surg J 2012; 32: 393-412.

8. Marten TJ. High SMAS facelift: combined single flap lifting of the jawline, cheek, and midface. Clin Plast Surg 2008; 35: 569-603, vi.

9. Baker DC. Lateral SMASectomy. Plast Reconstr Surg 1997; 100: 509-513. 
10. Marten TJ, editor. Facelift-state of the art. Seminars in plastic surgery. New York: Thieme Medical Publishing 2002.

11. Gamble WB, Manson PN, Smith GE, Hamra ST. Comparison of skin-tissue tensions using the composite and the subcutaneous rhytidectomy techniques. Ann Plast Surg 1995; 35: 447-453; discussion 453-444.

12. Becker FF, Bassichis BA. Deep-plane face-lift vs superficial muscle aponeurotic system plication facelift: a comparative study. Arch Facial Plast Surg 2004; 6: 8-13. doi: 10.1001/archfaci.6.1.8.

13. Mohammadi S, Ahmadi A, Salem MM et al. A comparison between two methods of face-lift surgery in nine cadavers: SMAS (superficial muscleaponeurotic system) versus MACS (minimal access cranial suspension). Aesthet Plast Surg 2015; 39: 680-685. doi: 10.1007/s00266-015-0543-3.

14. Verpaele A, Tonnard P, Gaia S et al. The third suture in MACS-lifting: making midface-lifting simple and safe. J Plast Reconstr Aesthet Surg 2007; 60: 1287-1295. doi: 10.1016/j.bjps.2006.12.012.

15. Verpaele A, Tonnard P. Lower third of the face: indications and limitations of the minimal access cranial suspension lift. Clin Plast Surg 2008; 35: 645659, vii. doi: 10.1016/j.cps.2008.04.001.

16. Prado A, Andrades P, Danilla S et al. A clinical retrospective study comparing two short-scar face lifts: minimal access cranial suspension versus lateral SMASectomy. Plast Reconstr Surg 2006; 117: 14131425; discussion 1426-1417. doi: 10.1097/01. prs.0000207402.53411.1e.

17. Tonnard PL, Verpaele A, Gaia S. Optimizing results from minimal access cranial suspension lifting (MACSlift). Aesthet Plast Surg 2005; 29: 213-220; discussion 221. doi: 10.1007/s00266-005-0047-7.
18. Mast BA. Advantages and limitations of the MACS lift for facial rejuvenation. Ann Plast Surg 2014; 72: S139-143. doi: 10.1097/sap.0000000000000092.

19. Gordon NA, Adam SI, 3rd. Deep plane face lifting for midface rejuvenation. Clin Plast Surg 2015; 42: 129142. doi: 10.1016/j.cps.2014.08.009.

20. Parikh SS, Jacono AA. Deep-plane face-lift as an alternative in the smoking patient. Arch Facial Plast Surg 2011; 13: 283-285. doi: 10.1001/archfacial.2011.39.

21. Marcus BC. Rhytidectomy: current concepts, controversies and the state of the art. Curr Op Otolaryngol head Neck Surg 2012; 20: 262-266. doi: 10.1097/MOO.0b013e328355b175.

22. Lemmon ML. Color atlas of SMAS rhytidectomy. First ed. Michigan: Thieme Medical Publisher; 1993

23. Stuzin JM, Baker TJ, Gordon HL, Baker TM. Extended SMAS dissection as an approach to midface rejuvenation. Clin Plast Surg 1995; 22: 295-311.

24. Lindsey JT. Five year retrospective review of the extended SMAS: critical landmarks and technical refinements. Ann Plast Surg 2009; 62: 492-496. doi: 10.1097/ SAP.0b013e31818ba77d.

Conflicto de intereses: Los autores declaran no tener conflicto de intereses.

Correspondencia:

Dr. David R. De-Rungs-Brown

American British Cowdray Medical Center Av. Carlos Fernández Graef Núm. 154-320,

Col. Santa Fe Cuajimalpa, 05300,

Cuajimalpa, Ciudad de México, México.

E-mail: de_rungs@hotmail.com 